\title{
Conocimiento, aprendizaje y capital social como motor de desarrollo
}

Fernando Chaparro, Ph.D.

Secretario Ejecutivo

Foro Global de Investigación Agropecuaria (GFAR) FAO, Roma.

\section{Resumen}

Este paper analiza la aparición de la Sociedad del Conocimiento como una consecuencia del doble impacto del gran avanzo científico que caracteriza esta virada de siglo, el reciente surgimiento de tecnologías de información y comunicación, y el proceso de globalización que está transformando los mercados de producción y finanzas, y causando profundos cambios en los ambientes institucionales de la generación del concocimiento y su utilización. Se analiza también la importancia de redes y procesos de aprendizaje; la sinergia y tensiones que son simultáneamente generadas por la dinámica interacción que está ocurriendo entre la "apropiación social y privada del conocimiento"; y los retos en la formación de nueva estructura normativa para la generación y utilización del conocimiento en la solución de cuestiones de política de derechos de propiedad intelectual. Se hace referencia también a casos innovadores de promoción de redes de aprendizaje y desarrollo de diálogo entre depositarios. Se relatan también experiencias en foros sobre cuestiones estratégicas y otros mecanismos relacionados a la apropiación social del conocimiento, así como el surgimiento del Global Forum on Agricultural Research (GFAR), como una nueva expresión de globalización de la ciencia.

\section{Palabras clave}

Sociedad del conocimiento; Globalización; Apropiación del conocimiento; Redes de aprendizaje; Propiedad intelectual; Internet.

\section{Knowledge, learning and social capital as key development factors}

\begin{abstract}
This paper analyses the emergence of the Knowledge Society as a consequence of the combined impact of the high rate of scientific progress that characterises this turn of century, the recent breakthroughs in information and communication technologies, and the globalisation process that is transforming production and finance markets and producing profound changes in the institutional environment of knowledge-generation and utilisation. The paper analyses the importance of learning networks and processes; the synergisms and tensions that are simultaneously generated by the dynamic interaction that is taking place between the "social and private appropriation of knowledge"; and the challenges in developing a new normative framework for knowledge generation and utilisation in solving policy issues related to intellectual property rights. Reference is also made to innovative cases of promoting learning networks and developing a dialogue among stakeholders. Experiences are also mentioned about fora on strategic issues and other mechanisms related to the social appropriation of knowledge, as well as the emerging of the Global Forum on Agricultural Research (GFAR), as a new expression of the globalisation of science.
\end{abstract}

\section{Keywords}

Knowledge society; Globalization; Knowledge apropriation ; Networks of learning; Intelectual property; Internet.

\section{CONOCIMIENTO Y SOCIEDAD}

En cada época histórica las sociedades humanas tienen rasgos o elementos que caracterizan la base misma de su organización social. Estos mismos elementos pueden también encontrarse en las sociedades humanas en muchas otras épocas y momentos de la historia. Sin embargo, cuando dichos elementos sociales se convierten en principios de acción, o en principios organizadores del comportamiento humano y de la forma como las organizaciones y las instituciones sociales funcionan y responden a su entorno, estos elementos se convierten en rasgos que caracterizan el entorno social y la época histórica.

Ese es el papel que el conocimiento desempeña en el mundo en este inicio del siglo XXI. Toda sociedad humana siempre ha generado conocimiento sobre su entorno y sobre su realidad, física y social. Es un aspecto que surge de la necesidad misma que tiene el hombre de tratar de explicar o comprender el medio que habita. Pero en las formas de organización social que están actualmente surgiendo, el conocimiento comienza a tomar una dimensión, y a desempeñar un papel en la sociedad, que va más allá del papel que históricamente ha siempre cumplido. En las sociedades que se perfilan para el tercer milenio, la posición de cada persona en la sociedad crecientemente es el producto del conocimiento que él o ella han logrado desarrollar o construir. La sociedad del mañana se vislumbra como una sociedad en la cual cada individuo y cada organización construirá su propia capacidad de acción, y por lo tanto su posición en la sociedad, a través de un proceso de adquisición y desarrollo de conocimiento, y de la consolidación de su capacidad para generar nuevo conocimiento, que le permita adaptarse dinámicamente a una realidad en rápido proceso de cambio y transformación.

Lo anterior solo se logra a través de procesos continuos de generación y uso del conocimiento, organizados de tal forma que puedan contribuir a procesos de aprendizaje social. Este es el rasgo central de las Sociedades del Conocimiento. Estas últimas son sociedades con capacidad para generar conocimiento sobre su realidad y su entorno, y con capacidad para utilizar dicho conocimiento en el proceso de concebir, forjar y construir 
su futuro. Es decir, de utilizarlo en el proceso permanente de construcción de la sociedad, a través del desarrollo, transformación permanente y consolidación de sus principales instituciones sociales. De esta forma, el conocimiento se convierte no solo en instrumento para explicar y comprender la realidad, sino también en motor de desarrollo y en factor dinamizador del cambio social.

\section{INFORMACIÓN, GLOBALIZACIÓN Y PROGRESO CIENTÍFICO}

Veamos cuáles son algunos de los principales factores que están incidiendo en la relación entre conocimiento y sociedad, y en definir las principales características del entorno en el que operamos. En los albores del Tercer Milenio el principal desafío que confrontamos es el de cómo desarrollar en las sociedades Latinoamericanas y del Caribe una capacidad para proyectarse en un mundo en rápido proceso de cambio, dominado por tres tendencias dinámicas y complejas que lo están profundamente transformando: el desarrollo de la sociedad de la información, la globalización, y el progreso científico y tecnológico que se está dando en diversas áreas de la ciencia, procesos que están generando nuevas oportunidades, pero también claros desafíos.

Las tecnologías de la información y las comunicaciones están teniendo un profundo impacto en todos los sectores de la actividad humana, desde la producción, hasta la educación y los servicios de salud. La convergencia de tres áreas tecnológicas anteriormente diferenciadas, que son la informática (computadores), las telecomunicaciones y la transferencia y procesamiento de datos y de imágenes, ha llevado a profundos cambios en la producción de bienes y servicios en las sociedades contemporáneas. La capacidad de acceso y de uso de dichas tecnologías, como son la microelectrónica, las diversas tecnologías relacionada con la multimedia, y la multiplicidad de aplicaciones que han surgido en la educación, la salud, la producción, los servicios y el control ambiental, están dando lugar a las llamadas sociedades de la información. La terciarización de la economía, inclusive en el sector agropecuario y manufacturero, es uno de los aspectos estrechamente vinculados a la informatización de la sociedad. ${ }^{1}$

La revolución producida por las tecnologías de la información y las comunicaciones ha generado, por primera vez en la historia de la humanidad, la posibilidad de movernos en la dirección de una verdadera sociedad global, basada en la capacidad para interactuar en tiempo real entre personas y grupos geográficamente dispersos, a través de Internet o del correo electrónico. La globalización es un proceso que va más allá de la apertura de mercados y de la internacionalización de la economía. Responde al proceso que Arnold Toynbee en su magna obra, "A Study of History", planteó como uno de los principales desafíos de la humanidad en este fin de siglo: la construcción de una sociedad global, que requiere una institucionalidad y un marco normativo diferente al del estado-nación, y al de los mecanismos intergubernamentales que han históricamente dominado el ámbito internacional. Se trata del surgimiento de la sociedad civil transnacional, que se refleja en la globalización de los mercados, de la producción, de los sistemas financieros, de la diversión y de la misma ciencia.

Es importante resaltar el hecho que la globalización no solo toca a aquellos que participan en este nuevo entorno. Incide así mismo en los sectores sociales que no tienen la posibilidad de insertarse y participar activamente en ella, pero que sufren sus consecuencias a través de procesos que esta última genera, como es el caso de la reestructuración competitiva, de cambios en los mercados de trabajo, o de procesos de transformación institucional que de ella se derivan. Los procesos de exclusión social que esto genera, pueden llegar a tener un impacto más significativo que los procesos de participación que dicho entorno facilita.

El tercer factor que domina este fin de siglo lo constituye el alto ritmo de progreso científico y tecnológico, y la relación cada vez más estrecha entre el conocimiento que el adelanto científico genera, y las múltiples aplicaciones del mismo en todos los sectores de la actividad humana. Las plantas transgénicas, los biopesticidas, las nuevas vacunas y drogas que están surgiendo, y los demás productos que la revolución de la biología molecular, la microelectrónica y los nuevos materiales están produciendo, abren nuevas oportunidades de crecimiento y de desarrollo. Al mismo tiempo, el alto ritmo de cambio científico también encierra grandes desafíos y peligros de desigualdad creciente, por medio de procesos de exclusión social con base en la capacidad de acceso a, y uso de, estas tecnologías.

La revolución científica que se está produciendo alrededor nuestro en este fin de siglo está generando una gran dinámica en las llamadas "nuevas áreas de la ciencia", o "nuevas tecnologías". Estrechamente relacionado con la dinámica de generación de conocimiento que se puede observar en ellas, estas nuevas áreas tienen tres características importantes. En primer lugar, están generando tecnologías genéricas con múltiples aplicaciones 
en casi todos los sectores de la sociedad: producción, educación, salud, servicios, diversión, manejo ambiental, funcionamiento del Estado, y otros. En segundo lugar, la distancia entre el conocimiento básico y su aplicación en la producción de bienes y servicios, es cada vez menor. Los procesos de producción, así como el suministro de servicios o el manejo de recursos naturales y de la biodiversidad, son cada vez más intensivos en ciencia, y por lo tanto en conocimiento. La distancia entre el laboratorio y la empresa se ha significativamente reducido, fenómeno que se puede observar en el alto número de empresas intensivas en ciencia que se crean semanalmente en las universidades de los países desarrollados, proceso cuya importancia se ha claramente incrementado. Esta característica tiene importantes implicaciones para el proceso de apropiación del conocimiento que se está dando en estas áreas científicas, sobretodo en lo referente a la relación entre apropiación social y apropiación privada del conocimiento, aspecto sobre el cual regresaremos posteriormente.

En tercer lugar, el ritmo del progreso científico en estas nuevas áreas de la ciencia se ha acelerado enormemente, llevando a la necesidad de desarrollar nuevas formas de organización de la investigación con el fin de poder mantenerse al día y de desarrollar masas críticas de investigación en campos que están cambiando y avanzando a un ritmo vertiginoso. Las oportunidades y requerimientos generados por el nuevo entorno están llevando a nuevas formas de organización de la comunidad científica, como las de consorcios de investigación, nuevas formas de cooperación universidad/industria, redes electrónicas de investigación y otras formas asociativas, que buscan, a través de la cooperación, responder al desafío generado por el alto ritmo de cambio científico y los crecientes costos de la investigación que esto conlleva.

\section{CONOCIMIENTO Y CAMBIO SOCIAL: EL MARCO INSTITUCIONAL}

La estrecha relación que existe entre conocimiento y sociedad, está llevando a que los cambios que se están dando en la ciencia no se den en el vacío. En forma paralela a los cambios en la ciencia, y a través de relaciones de causalidad complejas e interactivas, se están produciendo profundos cambios en las principales instituciones sociales, tanto en América Latina y el Caribe como en el resto del mundo. El papel del Estado, así como la relación entre éste y la Sociedad Civil, están en claro proceso de transformación. El proceso de apertura de mercados y de globalización, y los niveles de competitividad que se requieren para sobrevivir en este ámbito, conllevan la necesidad de reestructurar el aparato productivo en casi todos los sectores. Lo anterior se está reflejando dramáticamente en recientes cambios que se están produciendo en el sector agropecuario y manufacturero en todos nuestros países. A su vez, dichos procesos están teniendo un profundo impacto en el empleo, en los ingresos de la población, en la organización del trabajo y en el tipo de recursos humanos que el mercado laboral requiere. Desde el punto de vista de los recursos humanos que el nuevo entorno requiere, los anteriores cambios están poniendo una gran presión sobre el sistema de educación, en todos sus niveles. Debido a la importancia del conocimiento y la educación en las sociedades contemporáneas, la necesidad de repensar el sistema de educación se ha convertido en uno de los principales desafíos de las sociedades contemporáneas en este inicio de siglo.

Las ventajas comparativas de los países ya no reflejan ventajas basadas en la dotación de recursos naturales o en su ubicación geográfica. Por el contrario, depende de capacidades y ventajas adquiridas, con base en el desarrollo de recursos humanos y en la capacidad para generar y aplicar conocimiento, tanto en la producción como en la solución de problemas sociales. Estos últimos están llevando en muchos países a altos costos de transacción y a conflictos sociales crecientes, que limitan la competitividad real del sector productivo a pesar de los incrementos reales en productividad logrados por los adelantos científicos y tecnológicos. Dichas incongruencias llevan a lo que Fernando Fajnzylber en sus conocidos estudios realizados en la CEPAL llamó la "competitividad espúrea", que no es sostenible en el mediano y largo plazo. A su vez, estas incongruencias generadas por la falta de integración de consideraciones de equidad y de sostenibilidad llevan al deterioro de la competitividad, a la pérdida de empleo, y a círculos viciosos de creciente pobreza y niveles de conflicto social, y, lo que es más grave, de pérdida de legitimidad de instituciones sociales básicas. En este nuevo contexto es necesario abordar el problema de la competitividad de sectores de la producción o de la sociedad en forma integrada, ya que ella es estructural.

Todo lo anterior está llevando a profundas crisis en las principales instituciones sociales de los países de América Latina y el Caribe. El sistema político, el cambiante papel del Estado, las nuevas formas de intervención de éste, el precario equilibrio entre el mercado y la nueva normatividad emergente, los cambios en la organización del trabajo y en el mercado laboral, la presión sobre un sistema educativo obsoleto que no prepara los recursos humanos que el nuevo entorno requiere, la pérdida de 
competitividad y de empleo en muchos sectores de la producción, el deterioro del medio ambiente y de los recursos naturales, son los claros síntomas de la profunda crisis institucional que se confronta en muchas sociedades del mundo al inicio del nuevo siglo. Estas consideraciones están en el centro del debate que se está dando en toda la región, así como en Europa, en América del Norte y en el Asia, sobre el desafío que todos los países confrontan al iniciarse el Tercer Milenio, y sobre cómo lograr el desarrollo de sociedades del conocimiento que logren aprovechar las oportunidades que la ciencia y la tecnología ofrecen en la solución de estos problemas.

\section{CARACTERÍSTICAS CENTRALES DE LAS SOCIEDADES DEL CONOCIMIENTO}

Las anteriores consideraciones remiten al papel del intelectual en la sociedad, así como al papel de la investigación y de la universidad en la construcción de la sociedad que deseamos para el futuro. Es decir, al papel que la ciencia y la tecnología, y por lo tanto el conocimiento, pueden jugar en la construcción de sociedades con capacidad para responder con éxito a los desafíos que se confrontan al inicio del tercer milenio.

Esto nos remite, así mismo, a los cuatro aspectos o procesos fundamentales que caracterizan a las sociedades del conocimiento. El primero se refiere a la importancia que tiene el conocimiento como el factor de crecimiento y de progreso más importante en las sociedades contemporáneas, y por lo tanto la educación como el proceso más crítico para asegurar el desarrollo de sociedades dinámicas, con capacidad de responder al nuevo entorno y de construir su futuro. Aquí nos referimos a una educación con capacidad para desarrollar creatividad en la persona y en la sociedad, a través del desarrollo de la capacidad analítica y de comprensión en el niño, y en el futuro ciudadano y profesional. Es decir, en una educación orientada a "aprender a aprender", y no en procesos de memorización. Lo anterior implica reformas profundas en el sistema de educación, como es el caso de la enseñanza de la ciencia y del pensamiento analítico en los diversos niveles del proceso educativo. Así mismo, requiere una acción muy decidida en la dirección de asegurar calidad y excelencia en la educación en los diversos niveles de este sistema, tanto en la educación básica como en la educación superior. Es interesante constatar que este desafío se está confrontando tanto en países desarrollados como en países en desarrollo.
La construcción de sociedades del conocimiento requiere, en segundo lugar, el desarrollo de procesos de apropiación social del conocimiento, por medio de los cuáles este último es apropiado por la sociedad, o por actores o sectores específicos de la misma. Esta apropiación se da por parte de individuos, de organizaciones (como es el caso de empresas), o de la comunidad y de las instituciones sociales básicas de la sociedad. A través de dicho proceso, el conocimiento se convierte en "bienes públicos" que, al acumularse e interrelacionarse, pasan a formar parte del capital social con el que cuenta una empresa, una organización, una comunidad, una institución social, o la sociedad misma. Es sobre la base de este conocimiento socializado, o capital social, que las organizaciones y las instituciones sociales pueden responder a las oportunidades y los desafíos que el nuevo entorno brinda, y adaptarse a cambios rápidos que en este mismo se presentan.

El anterior proceso lleva a un tercer factor importante, que constituye una de las principales dimensiones de las sociedades del conocimiento: Los procesos de aprendizaje social, por medio del cual el conocimiento crea o fortalece capacidades y habilidades en las personas, comunidades y organizaciones que se lo apropian. Entendido de esta forma, el aprendizaje es el proceso fundamental que lleva del conocimiento a la innovación y al cambio social. El aprendizaje puede ser visto como un proceso de educación continua, que crea a su vez nuevo conocimiento. La capacidad de generar procesos dinámicos de aprendizaje social, a nivel de una organización, de la comunidad, o de instituciones sociales básicas, es el elemento más crítico de las sociedades del conocimiento. Los dos procesos complementarios de apropiación del conocimiento, de una parte, y de aprendizaje social, por la otra, llevan a una concepción dinámica de las relaciones que se dan entre el conocimiento, el sujeto que conoce, y el entorno sobre el cual el sujeto actúa con base en ese conocimiento.

Un último rasgo importante de las sociedades y de las organizaciones del conocimiento es el desarrollo de un pensamiento estratégico y prospectivo, necesario para comprender los cambios que se están produciendo en el entorno, proyectar tendencias dinámicas y posibles futuribles, e identificar aspectos críticos o estratégicos que permitan orientar los esfuerzos que se realizan en el proceso de generación de conocimiento y de cambio social o desarrollo organizacional, en una dirección adecuada. Este último factor facilita el uso del conocimiento como motor de desarrollo y como instrumento crítico en el proceso de "construcción de sociedad." 


\section{APROPIACIÓN SOCIAL DEL CONOCIMIENTO: FORMAS QUE TOMA Y TEMAS DE POLÍTICA}

Para que el conocimiento pueda convertirse en factor de crecimiento y de progreso, es necesario ir más allá del desarrollo de una capacidad para generarlo. Se requiere que este último sea interiorizado por parte de individuos, organizaciones, empresas e instituciones sociales. Es decir, que el conocimiento permée la sociedad. Es aquí donde surge la importancia del proceso de apropiación social y apropiación privada del conocimiento, como las dos principales formas por medio de las cuales el conocimiento se convierte en innovación.

En esta sección analizaremos las diversas formas que toma el proceso de apropiación del conocimiento y los medios a través de los cuáles se pueden inducir procesos de aprendizaje social. Estos dos procesos complementarios desempeñan un papel central para que el conocimiento pueda convertirse en factor de innovación, de cambio social y de progreso. Así mismo, se identificarán algunas de las principales preguntas de política ("policy issues") que esto genera.

\section{a) Apropiación Social y Apropiación Privada del Conocimiento}

Cuando la investigación y el desarrollo tecnológico generan bienes públicos, o conocimientos que pueden convertirse en bienes públicos, estos últimos pueden ser libremente utilizados por la sociedad o por aquellos sectores sociales interesados en ellos. Es aquí cuando se da el proceso de apropiación social del conocimiento. Esto es cierto tanto en el caso de las ciencias sociales, como en el caso de las ciencias básicas o en el caso de la producción. Mientras el primer caso remite a la creación de "lo público", tema sobre el cual regresaremos, el segundo caso remite al desarrollo de "capacidades tecnológicas" que se construyen en un sector o en una comunidad específica, basadas en el conocimiento acumulado sobre recursos naturales, sobre biodiversidad, o sobre tecnologías de producción. Aqui nos referimos tanto a conocimiento científico generado por la investigación como al conocimiento empírico tradicional que una comunidad acumula. Estas dos modalidades forman parte del capital social que una sociedad desarrolla, reflejando su conocimiento sobre diversos temas o aspectos de su entorno.

Con la creciente importancia del papel que desempeña el sector privado en la investigación científica y tecnológica, está creciendo en relevancia otra forma de apropiación de los resultados de la investigación: la apropiación privada del conocimiento. Esta última se basa en una dimensión muy diferente: las tecnologías apropiables, que desempeñan un papel muy importante en diversos sectores de la producción. Las tecnologías apropiables han desempeñado un papel dinamizador del cambio técnico, ya que ellas representan una de las motivaciones más fuertes para que el sector privado invierta en investigación y desarrollo tecnológico. Por lo tanto, la dinámica de las tecnologías apropiables se han constituido en uno de los principales motores del cambio técnico.

Sin embargo, el manejo adecuado de la apropiación privada del conocimiento también plantea desafíos en ciertos sectores o temas en los cuáles el conocimiento público ha tradicionalmente desempeñado un papel crítico desde el punto de vista del desarrollo equitativo y sostenible de un país. En este caso la creciente importancia de las tecnologías apropiables puede llevar a sesgos hacia productos de alta rentabilidad, mientras que las áreas científicas y de la producción que responden a necesidades del desarrollo social (v.gr. producción de alimentos, manejo de biodiversidad, desarrollo sostenible) pueden perder importancia. Es aquí donde comienzan a perfilarse posibles conflictos entre la racionalidad pública y la racionalidad privada en el proceso de apropiación del conocimiento. El principal desafío que se confronta es el de cómo mantener un equilibrio dinámico entre estos dos principios de apropiación, debido al hecho que cada uno desempeña un papel importante en la dinámica del cambio tecnológico y en el desarrollo equitativo y sostenible de las sociedades contemporáneas.

\section{b) Cambios en la Ciencia y en los Procesos de Apropiación del Conocimiento}

Es importante destacar que las formas de apropiación del conocimiento están cambiando muy rápidamente, como consecuencia de los cambios que se están produciendo en la ciencia misma como forma de conocimiento, o en la ciencia como institución social. La relación entre apropiación social y apropiación privada del conocimiento, está significativamente cambiando en varios sectores como consecuencia de la creciente importancia que tienen las "tecnologías apropiables" ("proprietory technologies") en las nuevas áreas de la ciencia. Por ejemplo, como consecuencia de los cambios que se están produciendo en la revolución de la moderna biología molecular, el proceso de privatización está crecientemente involucrando resultados generados por la investigación básica (v.gr. conocimiento genético), que hasta hace poco era del dominio público. ${ }^{2}$ Esto se debe al hecho ya observado anteriormente, de que la distancia entre investigación 
básica y su aplicación en la producción de bienes y servicios es cada vez menor. La distancia entre el laboratorio y la empresa se ha significativamente reducido.

La profundidad de los cambios que se están dando puede constatarse en el hecho que en los últimos siete a diez años el ámbito institucional de la investigación en biología molecular ha cambiado significativamente. Pasó de ser un campo de investigación en el que predominaban las universidades y el sector público, a uno en el que más del 80\% de la investigación en biología molecular la realiza o la financia el sector privado (estas son cifras a nivel global). En este mismo período la inversión pública en este campo ha permanecido relativamente estable mientras que la inversión privada se incrementó considerablemente, llegando a jugar el papel predominante que se refleja en las cifras anteriormente mencionadas.

Estos profundos cambios tienen implicaciones contrastantes. Por un lado, han claramente acelerado la generación de conocimiento y el ritmo de progreso científico. Al mismo tiempo, estos mismos cambios simultáneamente generan obstáculos al acceso del conocimiento, así como complejos problemas éticos y legales como consecuencia del proceso de apropiación privada (patentamiento) de éste. Existe una clara tensión dialéctica entre aquellos factores del nuevo entorno que generan limitaciones a la circulación libre del conocimiento y al acceso a dichas tecnologías, y los factores de ese mismo entorno que están acelerando el ritmo de generación y de circulación de conocimiento a través de la revolución de la informática y las telecomunicaciones y del proceso de globalización de la ciencia que estamos confrontando. Mientras esta última estimula la aparición de diversas y novedosas formas de cooperación y de intercambio de resultados de investigación, la privatización tiende a limitar el acceso a este último y su libre circulación. Lo anterior genera una tensión dinámica entre estas dos tendencias, que puede derivar en tensiones conflictivas en ciertos casos. El manejo de estos factores complejos, que simultáneamente inducen a la cooperación y a la globalización, por un lado, pero que al mismo tiempo limitan y restringen la circulación del conocimiento, por el otro, es uno de los desafíos que se confrontan en la consolidación de la nueva ciencia mundial.

Una de las manifestaciones que la privatización puede generar son los mayores obstáculos que se levantan a la transferencia de tecnología entre países, y especialmente hacia los países en desarrollo. Las políticas de los países industrializados utilizan ahora más explícitamente el conocimiento y la tecnología como armas de competencia comercial, y por lo tanto, buscan ganancias rápidas en el mercado internacional, que pueden encarecer las nuevas tecnologías o simplemente excluir del acceso a las mismas a potenciales compradores.

Una segunda consecuencia del proceso de privatización es la de la creciente importancia que está tomando el tema de la propiedad intelectual, en un contexto donde el marco normativo todavía está en proceso de definición. La propiedad de los resultados y productos que se obtienen a través de la biología molecular son más complejos de determinar que los tradicionales problemas de patentes industriales. Prueba de esto es que el nuevo marco normativo, que está todavía en proceso de consolidación, está surgiendo de litigios entre multinacionales que hacen investigación en este campo, y que se demandan mutuamente con el fin de ir definiendo el nuevo marco normativo a partir de la doctrina jurídica que se determina en dichas sentencias. Los especialistas en las nuevas formas que está tomando la propiedad intelectual están jugando un papel de creciente importancia en este contexto.

Una tercera consecuencia del proceso de privatización del conocimiento es el de la importancia que toman las alianzas estratégicas con el sector privado, tanto nacional como multinacional, para desarrollar esfuerzos conjuntos y tener acceso a las nuevas tecnologías. Pero este enfoque implica desarrollar una capacidad para negociar tecnología, y para establecer alianzas estratégicas bajo condiciones favorables. La necesidad de desarrollar una capacidad local de investigación en biotecnología para poder negociar con éxito el acceso a las nuevas tecnologías que la moderna biología molecular está produciendo, se analiza en un reciente informe sobre las aplicaciones de la biotecnología en el sector agropecuario. ${ }^{3}$ La negociación de tecnología vuelve a retomar una gran vigencia, pero en forma diferente a la negociación que se realizaba en épocas anteriores. En el contexto actual se le da más peso a las alianzas estratégicas y a insertarse en redes de innovación en este campo, donde participan universidades, centros de investigación y empresas.

\section{c) Apropiación Social: La creación de "Lo Público" y Procesos de Aprendizaje Social}

En la sección anterior analizamos la tensión dialéctica entre apropiación social y apropiación privada del conocimiento, así como algunas de las preguntas de política (en el sentido de "policy issues") que esto genera. En esta sección pasamos a un nivel diferente del análisis 
del uso del conocimiento en las sociedades contemporáneas, que remite al papel de éste último en la creación de "Lo Público" y en el desarrollo de procesos de aprendizaje social. Para que los resultados de la investigación sobre temas de interés nacional y sobre el entorno socioeconómico se conviertan en conocimiento útil y en factor dinamizador del cambio social, se requiere de un proceso de apropiación social del mismo que pasa por la creación de espacios de debate público sobre estos temas. Esto contribuye a desarrollar y consolidar "lo público": la generación de bienes públicos de conocimiento que pueden contribuir al buen funcionamiento de la sociedad, a la formación de una opinión pública informada, a la formación del ciudadano, al fortalecimiento de la sociedad civil, y a incrementar la capacidad de una sociedad o de una comunidad de adaptarse a un entorno rápidamente cambiante.

Uno de los desafíos que aquí se confrontan es el de cómo aprovechar el proceso de generación y apropiación del conocimiento para inducir procesos dinámicos de aprendizaje social, en los actores que se apropian de él. Por aprendizaje social nos referimos aquí al proceso por medio del cual la apropiación y uso del conocimiento crean o fortalecen habilidades y capacidades en las personas, comunidades u organizaciones que se los apropian, convirtiéndose así en factor de cambio y de progreso en la sociedad, en sus instituciones y en las empresas del sector productivo.

Uno de los requisitos importantes para lograr lo anterior es el de desarrollar una estrategia orientada a integrar a los principales actores sociales al análisis y discusión de los problemas de su sociedad, ya sea a nivel nacional o al nivel de una institución o de una comunidad específica. Esto requiere generar un amplio proceso de participación en espacios públicos de debate sobre temas de interés nacional, buscando involucrar sectores amplios de la sociedad civil. Con el fin de lograr el objetivo de aprendizaje social que se busca, es necesario integrar a los empresarios, a los trabajadores y a los diversos usuarios del conocimiento a través de Foros de Discusión y de otros mecanismos, en los que estos últimos interactúan con investigadores y con intelectuales relacionados con los diversos temas bajo análisis.

Esta relación dinámica entre conocimiento y sociedad, especialmente cuando se logran procesos por medio de los cuales el conocimiento permea la sociedad, puede contribuir a desarrollar una sociedad participativa e innovadora, con capacidad de diálogo y de convivencia. Es decir, una sociedad donde este proceso contribuya a la formación del ciudadano, a la constitución de una opinión pública informada, a desarrollar mecanismos de resolución de conflictos basados en el respeto de opiniones diferentes en contextos multiculturales y pluri-actoriales, y en donde el conocimiento sobre su propia realidad y sobre la relación con su medio ambiente asegure un desarrollo equitativo y sostenible. Por este medio se puede contribuir a la construcción de la sociedad del conocimiento que el siglo XXI requiere.

Una de las dimensiones más importantes en el tema que estamos analizando es el de las experiencias que se han venido desarrollando en el campo de la investigación y la investigación-acción relacionada con la participación ciudadana. Experiencias interesantes pueden identificarse en diversas ciudades Latinoamericanas, como es el caso de Sao Paulo, de Bogotá y de México. En Europa y en el Asia se están desarrollando esfuerzos similares. En estas diversas experiencias hay dos actores que desempeñan una función especialmente importante: el gobierno local y el papel que juega la universidad en su comunidad inmediata. Uno de los desafíos que se confronta aquí es el de cómo sistematizar el conocimiento, a menudo personal y vivencial, que surge de estas experiencias, y convertirlo en un conocimiento extrapolable que se pueda convertir en una pedagogía de la participación, que promueva la convivencia y la cohesión en las sociedades contemporáneas.

\section{d) Apropiación del Conocimiento a través de Organizaciones que Aprenden}

Uno de los principales recursos con que cuenta un país son sus organizaciones. Estas últimas pueden ser organizaciones de producción (empresas), organizaciones académicas (universidades), organizaciones de investigación y desarrollo tecnológico (centros de investigación e institutos tecnológicos), organizaciones políticas (partidos políticos), u organizaciones en cualquier sector de actividad humana. Desde inicios de este siglo, Max Weber había ya identificado las organizaciones formales como una de las principales características de las sociedades contemporáneas. La mayor parte de los procesos de generación y aplicación del conocimiento se realizan en contextos organizacionales, en el seno de entidades de muy diversa índole. Así mismo, son organizaciones las responsables de una alta proporción de la producción, así como del suministro de servicios en cualquier sociedad contemporánea. La efectividad y eficiencia de las organizaciones determina, por lo tanto, la capacidad de la sociedad para producir, ser competitiva, satisfacer 
necesidades básicas y lograr la mayor parte de los objetivos que ella persigue.

Tomando en consideración su ubicuidad y sus múltiples funciones, el uso del conocimiento y la apropiación del mismo por parte de organizaciones representan una de las formas más importantes de apropiación del conocimiento en el mundo actual. Este proceso lleva a desarrollar y consolidar organizaciones con capacidad de aprendizaje (las llamadas "learning organizations"). Esto último responde a uno de los principales desafíos que tiene toda organización: asegurar su supervivencia en un medio cambiante, y para ello debe contar con la capacidad para adaptarse e integrarse creativamente e ese entorno, al mismo tiempo que crea nuevas realidades relacionadas con el logro de sus objetivos.

Para hacer esto, se necesita aprender continuamente con el fin de poder responder a los cambios que se producen en el entorno. Pero no se trata meramente de responder de manera adaptativa a dichos cambios, sino de desarrollar una capacidad para generar respuestas creativas y transformadoras, que trasciendan lo inmediato, y que le permitan a la organización recrear y construir su propio ambiente físico y social. ${ }^{4}$ Este es la característica central de las organizaciones que aprenden; el desarrollo de este tipo de organización desempeña un papel crítico en la creación de Sociedades del Conocimiento.

Uno de los desafíos que aquí se confronta es el de cómo convertir información y conocimiento personal, en conocimiento corporativo útil que incremente la efectividad de la organización, así como su capacidad de innovación y de respuesta creativa y transformadora. Lo anterior genera una capacidad de desarrollo y cambio organizacional, que son igualmente importantes en una empresa, en una universidad, o en un centro de investigaciones o de desarrollo tecnológico.

\section{e) Aspectos Eticos y Jurídicos en la Apropiación del Conocimiento}

En el proceso de uso y de apropiación social del conocimiento están surgiendo problemas éticos y jurídicos relacionados tanto con el uso del mismo, como con la propiedad intelectual. Con relación a los aspectos legales, la propiedad intelectual de los productos que se obtienen a través de la biotecnología y la informática, genera preguntas jurídicas más complejas que los problemas de patentes en otros campos de aplicación. Varios problemas están surgiendo. El primero tiene que ver con el hecho de que debido a la creciente importancia de las tecnologías apropiables (por el creciente valor económico del conocimiento), el proceso de apropiación privada se está extendiendo a áreas del conocimiento vinculadas a la investigación básica, que hasta hace poco eran de dominio público. Un segundo problema es de naturaleza más ética, y tiene que ver con aceptar la idea de una apropiación privada (patentada) del conocimiento sobre la estructura biológica de los seres vivos. Este problema se complica aún más cuando uno pasa del tema de plantas transgénicas al Genoma Humano. Detrás de la dimensión puramente legal de propiedad intelectual, surge la pregunta más fundamental de la manipulación de la vida a través del conocimiento científico, y las condiciones bajo las cuáles se debe desarrollar dicha actividad.

Hay una segunda dimensión de las preguntas éticas generadas por las nuevas áreas de la ciencia, relacionadas con el impacto que pueden tener algunas de las tecnologías producidas por los recientes avances en biología molecular. Nos referimos aquí al caso de las plantas transgénicas, con relación a las cuales han surgido preguntas sobre sus impactos potenciales en el medio ambiente, o inclusive en la salud humana. Esto lleva a una nueva función en rápida expansión en este campo, que es el de la Bioseguridad, donde apenas ahora estamos desarrollando una capacidad en los países de América Latina y el Caribe.

Una tercera dimensión de los problemas éticos y jurídicos que están surgiendo tiene que ver con la interacción entre el conocimiento tradicional, de comunidades indígenas y comunidades campesinas, y el conocimiento generado por la ciencia moderna. Al integrarse conocimiento tradicional en nuevo conocimiento que se produce a través de investigación científica, sobretodo en el campo de recursos genéticos y de sus diversos usos, se genera un proceso de apropiación social (y en ciertos casos privada) del conocimiento, que involucra el primero, integrándolo en el segundo. La valoración del conocimiento tradicional, y su interacción con otras formas de conocimiento y sobretodo con la ciencia moderna, es un aspecto al que tendremos que prestarle mayor atención en el próximo futuro. A nivel global, estos aspectos se están discutiendo en el contexto de las negociaciones internacionales que se están llevando a cabo en la Comisión de Recursos Genéticos para la Alimentación y la Agricultura, de la FAO, y en el contexto de la Convención de la Diversidad Biológica. 
f) Intermediación del Conocimiento y Gestión del Conocimiento: Dos Funciones Críticas de las Sociedades del Conocimiento

Uno de los principales desafíos que se confrontan en las diversas formas de apropiación del conocimiento que analizamos en las secciones anteriores es la de la intermediación o gestión del conocimiento. La importancia de la gestión del conocimiento se ha recientemente agudizado debido al hecho de que actualmente se confronta un exceso de información generado por los adelantos analizados al inicio de este artículo ("information glut"). Al mismo tiempo, y esto contrasta con lo anterior, hay muchos usuarios de dicha información que no saben donde encontrarla, o no pueden utilizarla en su estado original (información en bruto). Esta última debe ser analizada y organizada en forma tal que pueda convertirse en conocimiento utilizable por parte del usuario. Uno de los desafíos que aquí se confronta es el de convertir información en conocimiento útil, para usuarios específicos de dicho conocimiento. Esta función, que podemos llamar de intermediación del conocimiento ("knowledge brokerage"), añade un valor agregado de gran importancia para poder promover procesos de apropiación social y/o privada del mismo.

Surge aquí la necesidad de crear mecanismos de diversa índole que puedan procesar información, convirtiéndola en conocimiento útil, para usuarios específicos, sobre temas concretos, y suministrándola en tiempo real, facilitando así la movilización y la utilización de información y de conocimiento en procesos de toma de decisión, ya sea en el sector empresarial, en el sector gubernamental o en comunidades específicas (v.gr. a nivel municipal). Para responder a esta necesidad han venido surgiendo diversas organizaciones y experiencias piloto orientadas a desempeñar esta función en la sociedad. En este contexto, por ejemplo, comienzan a aparecer las primeras empresas del conocimiento, dedicadas exclusivamente a esta función.

La mayor parte de las empresas del conocimiento que han aparecido están orientadas a atender las necesidades del sector productivo y empresarial. A un nivel muy diferente, están surgiendo diversos mecanismos más adecuados a fomentar el proceso de apropiación social del conocimiento y a facilitar procesos de aprendizaje social, ya sea al nivel de una sociedad nacional o de una comunidad específica. Nos referimos aquí a las diversas experiencias que se están desarrollando en América Latina y el Caribe en la constitución de Foros Nacionales, a través de los cuáles se busca "socializar el conocimiento" sobre temas estratégicos de la agenda nacional o internacional. En
Brasil Joao Paulo dos Reis Velloso jugó un papel pionero en América Latina y el Caribe con la constitución del Foro Nacional de este país, sobre temas críticos para el desarrollo de la sociedad brasileña y su ubicación en un mundo globalizado. La experiencia del Brasil llevó, en la década de los noventa, a experiencias similares en Chile, en Colombia y en el Perú.

En el desarrollo de estas experiencias tanto a nivel micro (empresas del conocimiento), como a nivel macro (Foros Nacionales), uno de los desafíos que la sociedad de la información confronta es la del desarrollo y fortalecimiento de una capacidad de gestión del conocimiento ("knowledge management"). Esta función es de gran importancia para facilitar el desarrollo de organizaciones que aprenden, o para promover el cambio social en instituciones básicas de la sociedad. Un reciente informe de la OECD sobre las economías basadas en la información muestra claramente que la gestión y la intermediación (o distribución) de conocimiento, son el factor más importante para explicar la apropiación del mismo, la innovación y el empuje de los sectores más dinámicos que jalonan la economía. ${ }^{5}$

Este enfoque se refleja claramente en las estrategias corporativas de innovación que se pueden observar en muchas multinacionales en años recientes. Sobre el particular vale la pena mencionar un reciente análisis comparativo realizado en Europa sobre las diversas estrategias corporativas de innovación, que claramente demuestra que las empresas nacionales y multinacionales más exitosas no se concentran en innovación de productos o de procesos (donde se encuentra el conocimiento tecnológico más tradicional). Por el contrario, ellas se basan en forma creciente en gestión del conocimiento sobre mercados, sobre tendencias en los procesos de desarrollo tecnológico, sobre legislación que los influencian, o sobre otros factores que pueden incidir en su posición competitiva a nivel nacional y global. ${ }^{6}$ Es aquí donde se manifiesta realmente la sociedad del conocimiento en sus múltiples dimensiones. 
APROPIACIÓN SOCIAL DEL CONOCIMIENTO EN LA SOCIEDAD DE LA INFORMACIÓN: UTILIZACIÓN DE INTERNET Y DEL CIBERESPACIO

El proceso de apropiación social del conocimiento está tomando nuevas dimensiones en el contexto de las sociedades de la información, por las posibilidades y oportunidades que se abren a través del uso de las tecnologías de la información y las comunicaciones. En esta última sección haremos algunas reflexiones sobre el papel de internet y del ciberespacio en la construcción de la sociedad del conocimiento. Estas nuevas tecnologías y los espacios por ellas generados facilitan no solo la difusión del conocimiento, sino que tambien crean nuevas modalidades de participación tanto de la comunidad científica nacional como de sectores amplios de la población, en el análisis y discusión de temas de interés nacional, regional o global. Por lo tanto, estas nuevas tecnologías están en capacidad de hacer un aporte significativo a los procesos de apropiación social del conocimiento en las sociedades contemporáneas.

El ciberespacio es un poderoso medio no solo para intercambiar información, si no también para generar redes de conocimiento y redes de aprendizaje sobre temas de interés local y global. Las dos dimensiones de la interacción inter-personal, la interacción personal en el espacio real y la interacción electrónica en el ciberespacio, se complementan entre sí (no se remplazan ni compiten entre ellas). La posibilidad de utilizar internet y los nuevos espacios de participación que las tecnologías de la información facilitan, abren la posibilidad de procesos participativos más dinámicos y extensos, a lo largo y ancho de la sociedad nacional y de la sociedad global. Bien utilizadas, estas tecnologías facilitan procesos de apropiación social del conocimiento, de aprendizaje social y de creación de lo público, características esenciales de las sociedades del conocimiento.

Con el fin de destacar algunas de las experiencias recientes en la aplicación de estas ideas en iniciativas concretas relacionadas con la construcción de la sociedad del conocimiento, en esta sección haremos una muy breve referencia a dos ejemplos específicos. El primero es el de la experiencia en Colombia de un programa nacional orientado a desarrollar una mayor capacidad de generación y manejo del conocimiento en esta sociedad, a través del desarrollo de una agenda estratégica nacional utilizando el mecanismo de los Foros Nacionales mencionados en la sección anterior. El segundo, a nivel mundial, es un interesante caso de fortalecer la participación de los países en desarrollo en las formas cambiantes de la ciencia global en el sector agropecuario.

Entre las experiencias innovadoras que se han desarrollando en América Latina y el Caribe en lo referente al desarrollo de una agenda estratégica nacional utilizando los enfoques y mecanismos a los que hemos hecho referencia, vale la pena mencionar un programa que recientemente se abordó en Colombia bajo el título de Programa Conocimiento, Innovación y Construcción de Sociedad (CICS). El objetivo de este programa es el de desarrollar en Colombia una sociedad del conocimiento. Como se indicó al inicio de este artículo, las sociedades del conocimiento se caracterizan por su capacidad para generar conocimiento sobre su realidad y su entorno, y su capacidad parta utilizar dicho conocimiento en el proceso de concebir, forjar y construir su futuro. Lo anterior se está buscando lograr a través de la formulación y discusión de una agenda nacional que se está desarrollando con base en el análisis participativo de 10 temas estratégicos para el desarrollo del país. Para lograr lo anterior, este programa busca generar espacios de debate público sobre dichos temas a diversos niveles, que van desde el nacional hasta el local. Por este medio se busca fomentar procesos de apropiación social del conocimiento, así como procesos de aprendizaje social a partir de este último. En el desarrollo de estas ideas este programa está utilizando las nuevas tecnologías de la informática y las comunicaciones, así como las nuevas formas organizacionales de la comunidad científica asociadas a ellas. ${ }^{7}$

La Red del Programa CICS a través de la cual se está desarrollando esta iniciativa combina tres instrumentos complementarios entre si: (a) el uso selectivo de websites, (b) redes electrónicas de investigación y de reflexión, y (c) grupos de discusión en internet combinados con grupos que funcionan en el espacio real (v.gr. en la comunidad), buscando aprovechar la potencialidad de las nuevas tecnologías para generar espacios de debate sobre temas de interés nacional. En este enfoque, los websites no son solo plataformas pasivas de intercambio de información y de acceso a documentos. Por el contrario, al complementarse estos últimos con los foros temáticos, con las redes de investigación y con los grupos de discusión, los websites sirven de elementos facilitadores de diálogos dinámicos entre investigadores, y entre estos últimos y los usuarios de dicho conocimiento (el tipo de usuarios varía significativamente de un tema o otro). Esta visión dinámica del papel y de la potencialidad de las nuevas tecnologías de la información y las comunicaciones es la que permite utilizarlas en procesos de apropiación social del conocimiento y en procesos de aprendizaje social, 
a través de los cuales el conocimiento puede convertirse en instrumento de modernización y de cambio social.

El segundo ejemplo que se desea resaltar se relaciona con los profundos cambios que se están dando en cómo se hace ciencia a nivel global, lo que algunos observadores llaman "global science". En este contexto, las redes de investigación, sobretodo las redes electrónicas de investigación y de conocimiento que actúan en el ciberespacio, están desempeñando un papel crecientemente importante en el campo de la investigación pre-competitiva. ${ }^{8}$ Dos factores que inciden en la efectividad de las redes electrónicas como forma de organización de la investigación son, en primer lugar, la mayor velocidad de circulación del conocimiento entre expertos en un tema específico, y, en segundo lugar, la posibilidad de movilizar y combinar capacidades de investigación ubicadas en sitios e instituciones diferentes (cuando no existen los limitantes anteriormente mencionados). En forma creciente la generación de conocimiento es el fruto de redes nacionales y transnacionales de investigación y de innovación.

Estas redes también desempeñan un papel importante en la difusión e intermediación del conocimiento, y en la creación de espacios de debate en la sociedad sobre temas de interés local, nacional o global. En un informe de la Comunidad Europea sobre los desafíos que Europa confronta al iniciarse el siglo XXI, se destaca el hecho que el factor más importante que contribuye a fomentar procesos de apropiación del conocimiento y de innovación es el de poder integrar a personas, empresas, grupos e instituciones en "redes que colaboran, educan, capacitan y aprenden. Es decir, en redes de aprendizaje". ${ }^{9}$

Una de las iniciativas más interesantes que se enmarca dentro de este enfoque, es la del Foro Global de Investigación Agropecuaria (o GFAR como se lo conoce por sus siglas en inglés), que se estableció a fines de 1996 para facilitar este proceso en el ámbito de la investigación sobre biodiversidad, recursos naturales, investigación agropecuaria y desarrollo sostenible. La iniciativa del Foro Global busca complementar los esfuerzos de investigación y desarrollo tecnológico que se realiza en los centros internacionales del Grupo Consultivo de Investigación Agrícola Internacional (CGIAR), al integrar a estos esfuerzos a los demás actores (stakeholders) de la investigación en este campo.
La iniciativa del Foro Global (GFAR) se basa en la convicción de la creciente importancia que tienen la cooperación, las alianzas estratégicas entre centros de investigación y otros agentes del cambio tecnológico, y las redes transnacionales de investigación y desarrollo tecnológico, en el contexto del mundo globalizado que caracteriza este inicio de siglo. Con base en esto, el GFAR busca aplicar las ideas que se han discutido en este artículo con el fin de fortalecer la participación de los centros de investigación y de los diversos actores directos ("stakeholders") de la investigación agrícola y del desarrollo rural, en las nuevas formas de organización que están surgiendo en la ciencia mundial como consecuencia de la globalización de la ciencia y de la revolución de la informática. Estos últimos dos procesos están abriendo nuevas posibilidades de organización en la ciencia global, tanto a nivel regional como a nivel mundial. Se considera que la investigación internacional en temas agrícolas, y los desarrollos tecnológicos que de ella se derivan, ya no se realizan solamente en centros internacionales de investigación, sino también a través del esfuerzo mancomunado que realizan diferentes instituciones del orden nacional, regional e internacional, utilizando diversas formas y modalidades de cooperación y de acción conjunta. Los centros internacionales y regionales, que en el campo de la investigación agropecuaria han tradicionalmente desempeñado un destacado papel (por ejemplo en la llamada Revolución Verde), continuarán jugando un papel muy importante por la capacidad instalada que tienen, por la calidad de sus recursos humanos y por los bancos de germoplasma que administran. Pero las redes transnacionales de investigación y de innovación, así como las alianzas estratégicas entre centros de investigación y otros actores institucionales, tanto en países en desarrollo como en países desarrollados (centros de excelencia a nivel mundial, empresas, universidades, etc.), están surgiendo como una de las nuevas formas de organización de la comunidad científica a nivel regional y global. ${ }^{10}$

Diversos estudios realizados recientemente han claramente mostrado que tanto en el sector agropecuario como en el industrial, la innovación y el cambio tecnológico ya no dependen meramente de la acción de productores o de instituciones de investigación individuales, actuando de manera aislada. En forma creciente, la innovación es el resultado de procesos iterativos y de cooperación que se dan entre los diversos actores que intervienen en el proceso de generación y uso del conocimiento, dando lugar a lo que en este artículo hemos llamado redes de aprendizaje o redes de innovación. Estas son las piezas fundamentales sobre las cuáles están surgiendo las sociedades del conocimiento. 


\section{BIBLIOGRAFÍA}

Ang, J. y Pavri, F.: "A Survey and Critique of Impacts of Information Technology"; en: International Journal of Information Management, vol. 14, 1994, pp. 122-133.

Antonelli, C. (ed.): New Information Technology and Industrial Change: The Italian Case; Boston, Kluwer Academic Press, 1988.

Antonelli, C. (ed.): The Economics of Information Networks; Amsterdam, North Holland, 1992.

Bowden, D. y Blakemann, K.: IT Strategies for Information Management; London, Butterworths, 1990.

Braman, S. y Sreberny-Mohammadi (eds.): Globalization, Communication and Transnational Civil Society; London, Hampton Press, 1996.

Chaparro, F: "ICT, Networking and Knowledge Systems in Agriculture and Rural Development"; en: Proceedings of the Second Conference of the European Federation for Information Technology in Agriculture, Food and the Environment; Bonn, EFITA, Septiembre 1999.

Chaparro, F.: Conocimiento, Innovación y Construcción de Sociedad: Una Agenda para la Colombia del Siglo XXI; Bogotá, TM Editores, 1998.

Comisión Europea: Teaching and Learning: Towards the Learning Society; Bruxelles, Comisión Europea, 1997.

Cooper, C. (ed.): Information Technology Policy and National Economic Development; London, Routledge and Kegan Paul, 1998.

Dumort, A. y Dryden, J. (eds.): The Economics of the Information Society; Luxembourg, Office for Official Publications of the European Communities, 1997.

Dutton, W. H. (ed.): Information and Communication Technologies: Visions and Realities; Oxford, Oxford University Press, 1997.

Foray, D. y Lundvall, B.-A. (eds.): Employment and Growth in the Knowledge-Based Edconomy; Paris, OECD, 1996,

Gibbons, M., Limoges, C., Nowotny, H., Schwartzman, S., Scott, P. y Trow, M.: The New Production of Knowledge: The Dynamics of Science and Research in Contemporary Societies; London, Sage Publications, 1994.

Hirst, P. y Thompson, G.: Globalisation in Question; Cambridge, Polity Press, 1996.
Howitt, P. (ed.): The Implications of Knowledge-Based Growth for Micro-Economic Policies; Calgary, University of Calgary Press, 1996.

Howkins, J. y Valentin, R.: Development and the Information Age: Four Global Scenarios for the Future of Information and Communication Technology; Ottawa, IDRC, 1997.

IIASA: Proceedings of the International Workshop on the Global Science System in Transition; Vienna, IIASA, mayo 1997.

Lundvall, B.-A., y Johnson, B.: “The Learning Economy”; en: Journal of Industry Studies; vol. 1, No. 2, 1994, pp. 23-42.

Machlup, F.: The Production and Distribution of Knowledge in the United States; Princeton, Princeton University Press, 1962.

Mansell, R y When, U.: Knowledge Societies: Information Technology for Sustainable Development; Oxford, Oxford University Press, 1998.

Mansell, R. y Silverstone, R. (eds.): Communication by Design: The Politics of Information and Communication Technologies; Oxford, Oxford University Press, 1996.

Mitter, S. (ed.): The Cyber-Economy and the Developing World: The Question of Exclusion and Inclusion; London, Routledge and Kegan Paul, 1998.

OECD: The Knowledge Based Economy; Paris, OECD, OECD Development Centre, 1996.

Raman, K.S., y Yap, C.S.: "From a Resource Rich Country to an Information Rich Society: An Evaluation of Information Technology Policies in Malaysia"; en: Information Technology for Development; vol. 7, 1996, pp. 109-131.

Roche, E.M., y Blaine, M.J. (eds.): Information Technology, Development and Policy: Theoretical Perspectives and Practical Challenges; Aldershot, Avebury, 1996.

Schuler, D.: New Community Networks: Wired for Change; New York: Addison-Wesley, 1996.

Senker, J.: "Tacit Knowledge and Models of Innovation"; en: Industrial and Corporate Change, vol. 4, No. 2, 1995, pp.425-447.

Ziman, J.: Prometheus Bound: Science in a Dynamic Steady State; Cambridge, Cambridge University Press, 1994. 


\section{Conocimiento, aprendizaje y capital social como motor de desarrollo}

\section{NOTAS}

${ }^{1}$ Para un análisis del papel que las tecnologías de la información y las comunicaciones están desempeñando en la construcción de sociedades del conocimiento, con una capacidad de innovación, ver: Robin Mansell y Uta When (eds.): Knowledge Societies: Information Technology for Sustainable Development; London, Oxford University Press, 1998.

2 Sobre el particular ver Fernando Chaparro: Biotechnology Research Policy and the Role of Research Partnerships: The Case of Colombia; ponencia presentada en el Simposio Sobre Biotecnología y Bioseguridad, Washington, Banco Mundial, Septiembre de 1997.

${ }^{3}$ Ver CGIAR Private Sector Committee: Global Partnerships in Agricultural Research: A Private Sector Perspective; Washington, D.C., CGIAR Secretariat, 1997.

${ }^{4}$ Sobre este particular ver el análisis más detallado que se hace en el Informe de la Misión de Educación, Ciencia y Desarrollo: Colombia: Al Filo de la Oportunidad; Santafé de Bogotá, COLCIENCIAS/TM Editores, 1996, pp. 105-156.

${ }^{5}$ Ver Organización para la Cooperación Económica y el Desarrollo (OECD): The Knowledge-Based Economy; Paris, OECD/GD (96), 1996.

${ }^{6}$ Ver Ferdinando Chiaromonte: "Innovación para la Globalización en el Siglo XXI"; Ponencia presentada en el Seminario Internacional Sobre Innovación, Inversión y Riesgo, Medellín, PROANTIOQUIA y COLCIENCIAS, Julio 22-24 de 1998.
${ }^{7}$ Para mayor información sobre este programa, ver Fernando Chaparro: Conocimiento, Innovación y Construcción de Sociedad: Una Agenda para la Colombia del Siglo XXI; Santafé de Bogotá, TM Editores, Agosto de 1998.

${ }^{8}$ Sobre este particular, la Academia de Ciencias de New York realizó recientemente un análisis interesante de las características cambiantes de la ciencia global. Para un resumen de los resultados de este análisis ver Rodney Nichols: Proceedings of the Belagio Conference on Global Collaboration in Science and Technology; New York, New York Academy of Sciences, 1996. Así mismo, el IIASA de Viena también realizó un interesante análisis comparativo de las nuevas formas de organización de la ciencia global, sobretodo del papel que desempeñan las redes electrónicas de investigación. Ver IIASA: Proceedings of the International Workshop on The Global Science System in Transition; Vienna, IIASA, May 1997.

9 Ver Comisión Europea: Teaching and Learning: Towards the Learning Society; Bruselas, Comisión Europea, 1997, p. 33.

10 Para una descripción de la misión, los objetivos y las actividades que está comenzando a desarrollar el Foro Global de Investigación Agropecuaria, ver: "GFAR-2000 Conference: Highlights and Follow-up Action"; GFAR Secretariat, Roma, FAO, agosto 2000; y el informe presentado por la primera evaluación externa de los dos primeros años de funcionamiento de esta iniciativa: "The Global Forum on Agricultural Research (GFAR): First External Review"; Roma, GFAR Secretariat, octubre del 2000. 